Article

\title{
A Fuzzy Synthetic Evaluation Method of Flame Stability Based on Time-Frequency Analysis and Higher-Order Statistics
}

\author{
Haitao Zhang, Ming Zhou and Xudong Lan* \\ School of Aerospace Engineering, Tsinghua University, Beijing 100084, China; \\ zhanght15@mails.tsinghua.edu.cn (H.Z.); zmzlh@tsinghua.edu.cn (M.Z.) \\ * Correspondence: lanxd@mail.tsinghua.edu.cn; Tel.: +86-13810750789
}

Received: 15 January 2019; Accepted: 22 March 2019; Published: 27 March 2019

check for updates

\begin{abstract}
The flame combustion processes involves chemical reactions and therefore flame stability is difficult to accurately assess. Based on flame radiation measuring parameters, a new synthetic evaluation system of flame combustion stability is established. A series of combustion conditions with various fuel/air ratios is investigated. Flame radiation luminance fluctuating information is acquired on a low-cost flame detection device. Power spectrum and bi-spectral information of the phase domain are derived from time domain signals based on Fourier transform and higher order statistics based upon a de-noising algorithm. The time-frequency characteristics and the features of the bi-spectrum under various combustion conditions are qualitatively analyzed, and the simultaneous descriptive parameters from time, frequency, and phase domain are extracted. A theoretical model for comprehensive fuzzy evaluation has been constructed, and also an index system has been established. It is demonstrated that this judgment system is reasonable and effective. The results can be used as an analyzing tool for process engineers for improving combustion conditions.
\end{abstract}

Keywords: flame stability; frequency domain; bi-spectrum; fuzzy synthetic judgment

\section{Introduction}

Owing to the existential problems of frequent load variation and combustion regulation, as well as the generation of equipment faults, the boiler flame often deviates from the optimum working conditions, failing to ensure a stable and homogeneous flame along with intense and sufficient combustion. The deterioration in the stability of burning not only results in a decrease in thermal efficiency with more pollutants and noise production, but also can cause fire extinguishing or even induce explosions in certain extreme situations. Some theoretical and experimental research on combustion stability has been carried out. Yan et al. studied gas combustion characteristics and flame stability in a cone burner [1]. It was found that the cone burner was conducive to stable combustion of low calorific value burners. No flameout was observed at higher turbulence intensity. Moore et al. studied the flame stability of methane and oxygen [2]. Different forms of flame structure were obtained. The relationships between flame morphology and excess air coefficient, oxygen, and gas Reynolds number have been studied. Komarek and Polifke studied the flame dynamics under different swirl numbers [3]. The effect of flow fluctuation formed by swirls on flame stability were deeply analyzed by using the flame model established by heat release rate pulsation and velocity pulsation. Lilleberg et al. used different chemical reaction mechanisms to analyze the effect of gas equivalent ratio pulsation on combustion stability under different fuel flows [4]. Voigt and Habisreuther studied the influence of combustion heat release rate pulsation on vortex shedding [5]. The coupling relationship between vortex shedding and combustion stability in swirl combustion was analyzed and modeled. The stability 
of combustion has an important impact on the economy, environmental protection, and safety of boilers, thus making the evaluation of combustion stability extremely important. Effective evaluation of flame instability can direct operators to seek better standards as reference for real-time operation, so that the overall synthetic performance of the unit can be improved.

The flame radiates various types of energy signals during the combustion process, such as light, heat, and sound [6]. Modern flame detection technology can be divided into direct technology and indirect technology. Direct flame detection techniques include differential pressure technology and temperature technology. Although the principles of these technologies are simple, they are very sensitive to the noise on the site. Moreover, the parameters of flame detectors are also difficult to set. Indirect flame detection technologies are currently relatively advanced means of flame detection $[6,7]$. Flame detection based on flame radiation is the method employed most extensively at present but is quite limited in real application of an industrial flame due to the restriction of the theory $[8,9]$. The threshold setting of flame radiation intensity and flicker frequency are difficult to adapt to large range load variation, and detection self-adaptation is restricted as the angle of viewing field of the flame detection probe is in the narrow range [3]. Furthermore, the flame emission of a single burner is hard to distinguish, owing to the full-filling flame of the furnace, which lead to a serious peep phenomenon, namely the furnace flame is misjudged as the nozzle flame by the flame detection [10]. Much more, frequency selective filter circuits and relational components in detection devices, which influence the data transmission and the detection effect in the case of slight changes of the radiation signal, often perform poorly [6]. Besides, transmission quality is affected as the photoelectric detection unit and the signal amplifier are separated [8]. In the process of sampling the flame radiation signal, the main problem presented is to ensure that the sampling frequency meets the requirements of the data analysis. The flame flicker frequency is generally below $50 \mathrm{~Hz}$. The sampling frequency must be greater than $100 \mathrm{~Hz}$ according to the sampling theorem. It is guaranteed that the sampled signal will not be mixed [8]. Real-time flame detection on site is generally required to take no more than five seconds each time, including signal acquisition, analysis, and judgment.

Researchers and related R\&D companies have introduced a series of measurements. Zhou et al. presented a correlation detection method of flame combustion [11,12]. Two or more angled flame detection probes were used to detect a single burner flame, and the combustion state was determined based on the correlation analysis. A multi-band detection method was another advanced technique applied by Lv et al., which fused flame radiation signals of multi-bands to determine the flame state [13]. Detectors of some technical R\&D institutions exploit radiation luminance and flicker frequency to identification the flame state. Much research has been made in the area of extracting features of flame radiation signals. A combustion diagnosis method based on combined Fourier transform and mode identification was presented by Ma et al., in which the first lowest 30 signals of each flame's power spectrum were selected to be used as the neural network' s input signals [14]. Li et al. found that the flashing area of the fuel was often fluctuating, and the most prominent location for the flicker frequency was a function of fuel type, load size, fuel flow rate, primary wind speed, and burner diameter [8]. The flame detector developed by Huang et al. used an optical fiber and photoelectric conversion device to detect the flicker frequency and radiation intensity of visible light emitted by the flame [11]. Taking into account the installation angle and other issues, the position of the optical fiber was set at the secondary air outlet, avoiding the strong radiation and strong convection of the combustion flame. Ao et al. proposed a new method for distilling the signature by the wavelet algorithm; the signals were subject to a soft threshold value de-noising treatment with the pretreated information serving as a training input to the neural network [15]. A recent study by Chi et al. showed that four features in time and frequency domains were found to be effective for fuel tracking [16]. Fuzzy logic inference techniques were applied to combine different features together and infer the type of fuel being burnt. All these studies showed the current research of extraction of features frequently limited in the time domain and frequency domain. Research on the phase domain is very scarce. The phase domain 
can provide phase information of the digital signal. It can be seen that the traditional combustion evaluation method is limited in data analysis.

An experimental and measuring system for detecting flame combustion stability is constructed. It includes an optical sensing device, a transmission device, and a computer. The radiation signal is transmitted to the phototransistors of the probes The optical signal is converted to a voltage signal. The flame signal is obtained via A/D (Analog to Digital Converter) conversion. The data is transferred to the processing computer, and then the characteristic parameters from the time, frequency, and phase domain are extracted, respectively. The model of fuzzy comprehensive evaluation is established, and the index system is also established. As a comprehensive statistical analysis method, the fuzzy comprehensive evaluation method can be effectively used to reveal the flame combustion stability. The results of the fuzzy comprehensive evaluation are presented in the form of vectors, and the evaluation information provided is more abundant than other methods [17]. The fuzzy comprehensive evaluation method has strong applicability and can be used for comprehensive evaluation of subjective factors and objective factors [18]. Figure 1 shows the flowchart of flame detection.

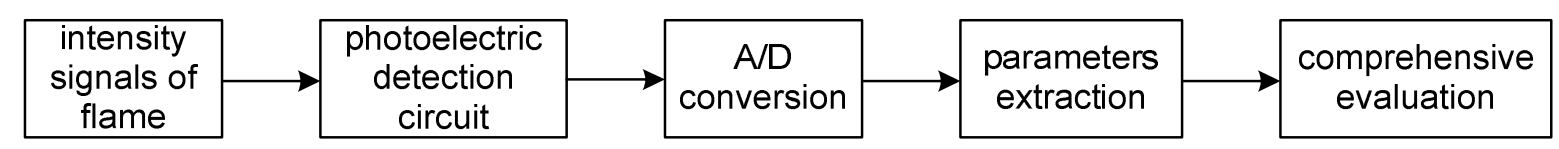

Figure 1. Flowchart of flame detection.

\section{Methods}

\subsection{Bi-Spectrum Analysis}

Power spectral analysis is a popular and efficacious method in traditional signal processing; however, phase information of signal is neglected in power spectral estimation [19]. Higher-order spectral analysis method is a new technology in signal processing in recent years. Higher-order spectral analysis based on higher-order cumulant not only preserves phase information of signals, but also restrains the interference of the effects of noise. These superior characteristics contribute to the wide range of applications in signal processing.

Bi-spectrum, the simplest analysis method of the HOS (Higher Order Statistics), appears as an indicator of non-linear transformations characterizing the signal. Bi-spectrum is always used to detect phase coupling. As a result, the bi-spectrum is particularly of use in detecting quadratic nonlinearity [20].

\subsubsection{Bi-Spectrum Definition and Phase Information}

Assuming $x(n)$ is a zero-mean-value and stationary random signal, and assuming the third-order cumulant $c_{k, x}\left(m_{1}, m_{2}, \ldots, m_{k-1}\right)$ is absolutely summable, that is,

$$
\sum_{m_{1}=-\infty}^{\infty} \sum_{m_{k-1}=-\infty}^{\infty}\left|c_{k, x}\left(m_{1}, m_{2}, \ldots, m_{k-1}\right)\right|<\infty
$$

then its $(k-1)$-dimensional Fourier transformation is defined by the $k$ th-order bi-spectrum:

$$
S_{k, x}\left(\omega_{1}, \omega_{2}, \ldots, \omega_{k-1}\right)=\sum_{m_{1}=-\infty}^{\infty} \ldots \sum_{m_{k-1}=-\infty}^{\infty} c_{k, x}\left(m_{1}, m_{2}, \ldots, m_{k-1}\right) \exp \left[-j \sum_{i=1}^{k-1} \omega_{i} m_{i}\right]
$$

The $k$ th-order bi-spectrum corresponds to $(k-1)$ bi-spectrum, such that the third-order bi-spectrum corresponds to bi-spectrum. Let $k=3$, then Equation (1) is simplified as bi-spectrum:

$$
B_{x}\left(\omega_{1}, \omega_{2}\right)=S_{3, x}\left(\omega_{1}, \omega_{2}\right)=\sum_{m_{1}=-\infty}^{\infty} \sum_{m_{2}=-\infty}^{\infty} c_{3, x}\left(m_{1}, m_{2}\right) \exp \left[-j\left(\omega_{1} m_{1}+\omega_{2} m_{2}\right)\right]=\left|B_{x}\left(\omega_{1}, \omega_{2}\right)\right| \exp \left(j \psi_{B}\left(\omega_{1}, \omega_{2}\right)\right)
$$


From the definition of the bi-spectrum in Equation (2), it follows that $B_{x}\left(\omega_{1}, \omega_{2}\right)$ is generally a plurality with a magnitude and phase, which reflects characteristic information of the signal better.

\subsubsection{Parameterized Bi-Spectral Estimation}

There are two chief approaches that have been used to estimate the bi-spectrum, namely, the nonparametric and the parametric approach, which is based on autoregressive (AR), moving average (MA), and auto-regressive moving-average (ARMA) models. The parametric approach not only has great estimation variance, but also increase computational cost. The ARMA model, which provides high resolution bi-spectral estimation and extracts phase information effectively, is a popular model in time-series analysis [21].

$$
x(n)=-\sum_{k=1}^{p} a(k) x(n-k)+\sum_{k=0}^{q} b(k) u(n-k)
$$

where $u(n)$ is assumed to be an independent identically distributed sequence, with variance $\sigma_{u}^{2}$. In practice, the observed process is noisy, that is,

$$
y(n)=x(n)+w(n)
$$

where noise process $w(n)$ is usually not known.

The determination of the model orders $p$ and $q$ is an important issue. Let $s(m)$ denote the singular values. If the cumulant estimates are perfect, we expect that exactly $p$ of the singular values will be nonzero. The AR order $p$ is then given by the value of $n$, which maximizes $s(n)-s(n+1)$, that is, it corresponds to the index at which the singular values show the maximum drop. MA order determination, which is based on estimating the theoretical variance of the sample estimates of $C_{3 y}(m, 0)$, is of equal importance. The sample estimates are asymptotically Gaussian and unbiased; hence, the threshold $t_{c}$ in

$$
\operatorname{Pr}\left\{\left|\hat{c}_{3 y}(m+1,0)\right| \leq t_{c}(m+1)\right\}=1-p f a
$$

is given by

$$
t_{c}(m+1)=\operatorname{erfinv}\left[(1-p f a) \sqrt{2 \sigma^{2}(m+1)}\right]
$$

where erfinv is the MATLAB inverse error function. Let $m_{0}$ denote the largest value of $m$ in the range $q_{\min }$ to $q_{\max }$ for which $\left|c_{3 y}(m+1,0)\right|>t_{c}(m+1)$, then the estimated order $q=m_{0}+1$; if such an $m$ does not exist, the MA order is declared to be $q_{\max }+1$.

Consider the residual time series obtained via,

$$
z(n)=\sum_{k=0}^{q} a(k) y(n-k)=\sum_{k=0}^{q} b(k) u(n-k)+\sum_{k=0}^{p} a(k) w(n-k)=\sum_{k=0}^{q} b(k) u(n-k)+\sum_{k=0}^{p} w_{1}(n)
$$

Routine ARMARTS (function name) uses the residual time series method to estimate the ARMA parameters-it estimates the AR parameters first, then computes the AR-compensated time series, and finally estimates the MA parameters. More details on Equations (1)-(8) can be found in references [19-21].

\subsection{Fuzzy Synthetic Evaluation}

Fuzzy synthetic evaluation quantifies ill-defined and hard-to-quantify factors on the application of the method of the compositional rule of inference. The fuzzy synthetic evaluation includes three steps:

(1) determining the factor set, the evaluation set, and the weight set;

(2) fuzzy evaluation of single factor; 
(3) synthetic evaluation.

\subsubsection{Establishment of Fuzzy Sets}

The combustion stability assessment model includes factor set, evaluation set, and weight set.

The factor set reflects the terms that affect the object:

$$
U=\left[u_{1}, u_{2} \ldots u_{i}\right]
$$

The assessment results are then categorized into some classes:

$$
V=\left[v_{1}, v_{2} \ldots v_{j}\right]
$$

where $v_{j}$ reflects the model condition, respectively. Then the evaluation grade is respectively given a value as:

$$
S=\left[s_{1}, s_{2} \ldots s_{j}\right]
$$

Considering the significant degree, the parameter is given a corresponding weight:

$$
A=\left[a_{1}, a_{2} \ldots a_{k}\right]
$$

where $a_{l} \geq 0(l=1,2 \ldots k)$ and $\sum_{l=1}^{k} a_{l}=1$. The distribution of weights is one of the most important factors that affect the assessment.

\subsubsection{Fuzzy Evaluation of a Single Factor}

For describing whether a factor belongs to a fuzzy set, a number between 0 and 1 is used to indicate the membership degree of the factor, rather than clear boundaries. Fuzzy evaluation is made from each factor to determine the object membership of the evaluation set. The evaluation result is expressed as:

$$
R=\left[\begin{array}{c}
R_{1} \\
R_{2} \\
\ldots \\
R_{j}
\end{array}\right]=\left[\begin{array}{llll}
r_{11} & r_{12} & \ldots & r_{1 j} \\
r_{21} & r_{22} & \ldots & r_{2 j} \\
\ldots & \ldots & \ldots & \ldots \\
r_{i 1} & r_{i 2} & \ldots & r_{i j}
\end{array}\right]
$$

where $r_{m n}$ is the membership of factor $u_{m}$ to evaluation $v_{n}$.

Membership function is the constitutional basis of dynamic fuzzy sets. The crucial procedure of fuzzy evaluation depends on the establishment of a realistic membership function. In the process of dynamic fuzzy evaluation, the membership function must follow five principles, namely threshold ability, differentiability, certainty of two poles, relativity of median, and rank preservation. In the process of determining the membership function, the principles respectively take on various roles and functions, determining the effectiveness of the membership function together.

\subsubsection{Synthetic Evaluation}

The fuzzy evaluation matrix can be obtained as:

$$
B=A \cdot R=\left(\begin{array}{llll}
a_{1} & a_{2} & \ldots & a_{n}
\end{array}\right) \cdot\left[\begin{array}{cccc}
r_{11} & r_{12} & \ldots & r_{1 j} \\
r_{21} & r_{22} & \ldots & r_{2 j} \\
\ldots & \ldots & \ldots & \ldots \\
r_{i 1} & r_{i 2} & \ldots & r_{i j}
\end{array}\right]
$$

where $B$ as the evaluation matrix represents the index of the fuzzy synthetic evaluation. 
For either combustion condition, a real index is obtained after the fuzzy synthetic evaluation.

$$
V=B \cdot S^{T}
$$

where $V$, as the evaluation score, reflects the evaluation of the actual case. More details on Equations (9)-(15) can be found in references [22,23].

\section{Experimental Setup and Process}

The flame detection system contains a data acquisition system, a gas burner, a fuel gas, a fan, flowmeters, and a DC power source.

\subsection{Signal Acquisition System}

In this experiment, the circuit system consisted of two parts: a flame detection circuit and a second-order filtering circuit. The optical signal conversion section adopted photosensitive transistors. In addition, the detection of background signals constructed an appropriate working condition for photosensitive conversion components, eliminated the direct current signals caused by background radiation, decreased the interference of the background glare from the outside, stopped the component from coming into the saturation area, and prevented the interference of the background glare from submerging useful signals; therefore, the dynamic extension of detection was broadened and the signal to noise ratio of the signals was increased. This made the quickly changing and effective dynamic flame signals much enhanced. Simultaneously, the reference source designed in the circuit provided the highly steady reference voltage of $+7 \mathrm{~V}$, and furthermore, strengthened the detecting effect of slight changes in flame. Figure 2 shows the signal acquisition system. The probe had a certain distance from the flame. Radiation from the flame did not affect the probe.

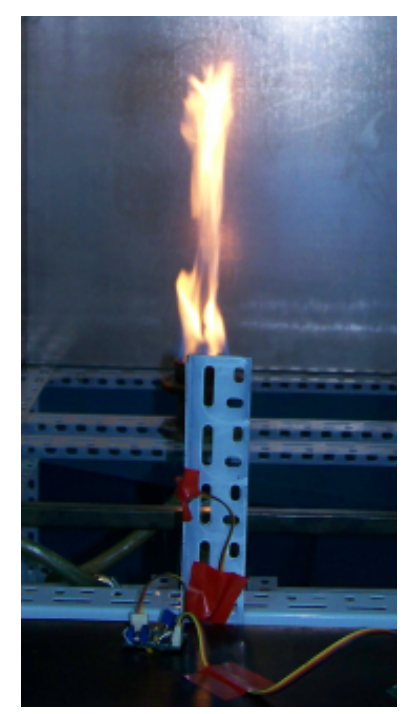

Figure 2. Signal acquisition system.

\subsection{Combustion Experimental System}

In the experiment, a suit of gas burning system that could control the flow of gas flux was designed. Air was inducted to the system by an axial flow fan, and then was divided into two parts: one part of the air (one-step air provision) was carried over by the gas flow to form premixed one-step air after the mixing to a certain extent, then entered the burner from the middle bottom of the nozzle, burst forth to burn and form a conform flame. The other part of the air (secondary air) entered directly into the bottom of the nozzle via a flowmeter, and subsequently flowed out from the annular flow pass of the gas vessel, then contributed to the air circumfluence diffusion burning. In the experiment 
the ration of secondary air was fixed at $2000 \mathrm{~L} / \mathrm{h}$, and the ratio of gas and one-step air provision was changed to accomplish a myriad of conditions. Due to the measuring range of the flowmeter, the ration of gas spanned from $40 \mathrm{~L} / \mathrm{h}$ to $600 \mathrm{~L} / \mathrm{h}$, while the ration of one-step air provision spanned from $0 \mathrm{~L} / \mathrm{h}$ to $1600 \mathrm{~L} / \mathrm{h}$. Along with the alteration of gas and one-step air, real-time flame intensity signals were collected and analyzed with MATLAB (Version 7.1.0.246 Service Pack 3, The MathWorks Inc., Natick, MA, USA).

\section{Characteristics Parameters Extraction}

\subsection{Time-Frequency Statistics}

During the combustion of fuel, a large amount of energy is radiated. On account of the altering of the flame shape, the thermal radiation, and the conversion between vibrational energy and rotational energy, the radiation intensity and the flicker frequency will be systolic. The stable flame means that the flame maintains a continuous envelope. For the unstable flame, the flame at the bottom of the burner fluctuates greatly, and the flame in the downstream flutters quickly. Six groups of time-frequency plots of radiant intensity signals in different working conditions are showed in Figure 3, among which the first threes are steady states, and the last three are unsteady ones. As is shown in Figure 3, there are large difference in time domain and frequency domain between steady states and unsteady states.

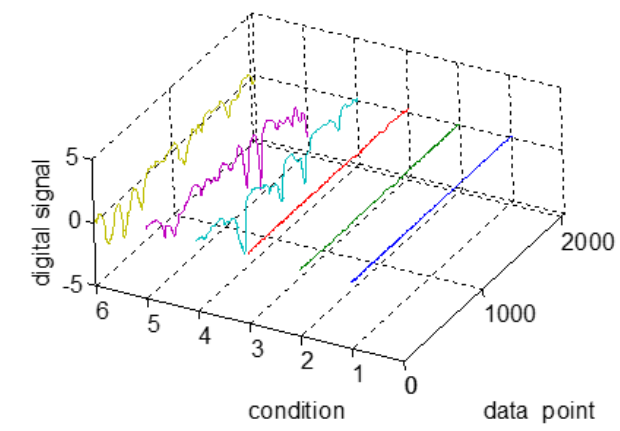

(a)

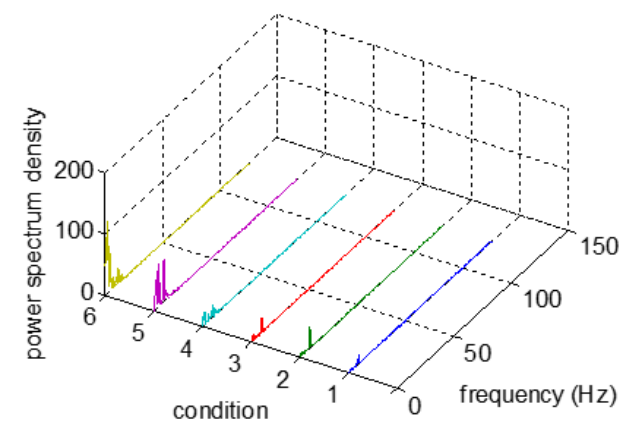

(b)

Figure 3. Time-frequency plot of radiant brightness of flame in typical working conditions: (a) time domain; (b) frequency domain.

The character of flame consists of dynamic components and stable components. The average value $I_{D C}$ and the fluctuating value $I_{A C}$ are used to show the intensity of the two states in the flicker signal of the flame. The average value and the fluctuating value can be expressed respectively as:

$$
\begin{gathered}
I_{D C}=\frac{1}{N} \sum_{n=1}^{N} I_{n} \\
I_{A C}=\sqrt{\frac{1}{N} \sum_{n=1}^{N}\left(I_{n}-I_{D C}\right)^{2}}
\end{gathered}
$$

where $N$ is the length of the sampling and $I_{n}$ is the $n$-th sample point of the signal.

During the process of processing the signals, the variance can measure the data pulsation. The larger variance reflects more fluctuation of the data. Merely based on the average value of the radiant intensity, the pulsating character of the radiant intensity cannot be reflected thoroughly. The specific variance can reflect the combustion stability [24]. It is defined as the variance of the dynamic components divided by the intensity value of the fluctuating value, expressed by mathematical formula as:

$$
v=\sum_{n=1} V_{n} / I_{A C n}
$$


where $V_{n}$ represents the variance of the dynamic components and $I_{A C n}$ represents the intensity value of the fluctuating components.

The flicker frequency reflects the radiation, pressure, and other features of the combustion flame. It is important to determine the most appropriate frequency of the flicker bi-spectrum, which consists of various frequency components. In the frequency domain, the weight average frequency is defined as flame flicker frequency. It describes the flame pulsating ability.

$$
F=\frac{\sum_{n=1}^{N}[P(n) \times f(n)]}{\sum_{n=1}^{N} P(n)}
$$

where $N$ is the frequency component number, $f(n)$ reflects the frequency of the $n$-th sampling point, and $P(n)$ means the power spectral density of the $n$-th sampling point. More explanations on Equations (17)-(20) can be seen in references $[25,26]$. Flame flicker frequency varies with the combustion conditions and reflects the internal factors and relationships, and consequently the combustion state by the monitoring and analysis of the flicker bi-spectrum. Flame flicker frequency is considered to reflect the stability of the combustion flame as an appropriate index. Owing to the spatial distribution and the interaction of the turbulence vortex, flicker state remains relatively stable statistically under certain conditions, and the distribution of the flame flicker frequency has certain characteristics [27]. When the flame combusts unstably, the intensity of the dynamic section increases, among whose low-frequency section increases noticeably. The distribution of the flame flicker frequency often serves at a lower level under instability conditions, while the distribution of the flicker frequency serves at a higher level under stability conditions. Figure 3 shows the similarly.

\subsection{Bi-Spectrum Analysis Statistics}

In the experiment, the pulsating signals of the radiant intensity of four working conditions were acquired: low-intensity steady state, high-intensity steady state, low-intensity unsteady state, and high-intensity unsteady state. The results of the bi-spectrum detection are presented in Figures 4-7, among which the upper row is the three-dimensional figure of the bi-spectrum. $|B x(f 1, f 2)|$ is the amplitude of the frequency bi-spectrum showing the mode of frequency coupling intuitively. The bottom row shows the contour plots corresponding to the upper row, from which the frequency distribution of the peak value can be observed. All the bi-spectrums utilized unitary coordinates, which defined the center of the frequency plane unfolded by $(f 1, f 2)$ as $(0,0)$; the neighbor of the center was a low frequency area, and the bi-spectrum showed the frequency coupling distribution of the peak values.

As is revealed in Figures 4-7, the existence of the amplitude in bi-spectrum explicated that the pulsating signals of the radiant intensity were non-linear and non-Gaussian signals.

From the bi-spectrum of steady state showed in Figures 4 and 5, the amplitudes in bi-spectrum of the low-intensity state were at relatively low levels, while the counterparts of the high-intensity state were relatively high. Quadratic phasic coupling occurred only in high frequency areas, due to the micro-scale fluctuation of radiation signals. With the accretion of the radiation intensity, the quadratic phasic coupling presented the phenomenon of range broadening and homogenization of distribution. There were no apparent distinctions between the bi-spectrums described in the low-intensity steady state and high-intensity steady state in Figures 4 and 5, but the amplitudes indicated that they were similar in the extent of non-linearity. Compared with the unsteady state (see Figures 6 and 7), the structure of frequency band was more various, indicating that the gas and air were mixed evenly, and the effect of frequency coupling was more distinct. 

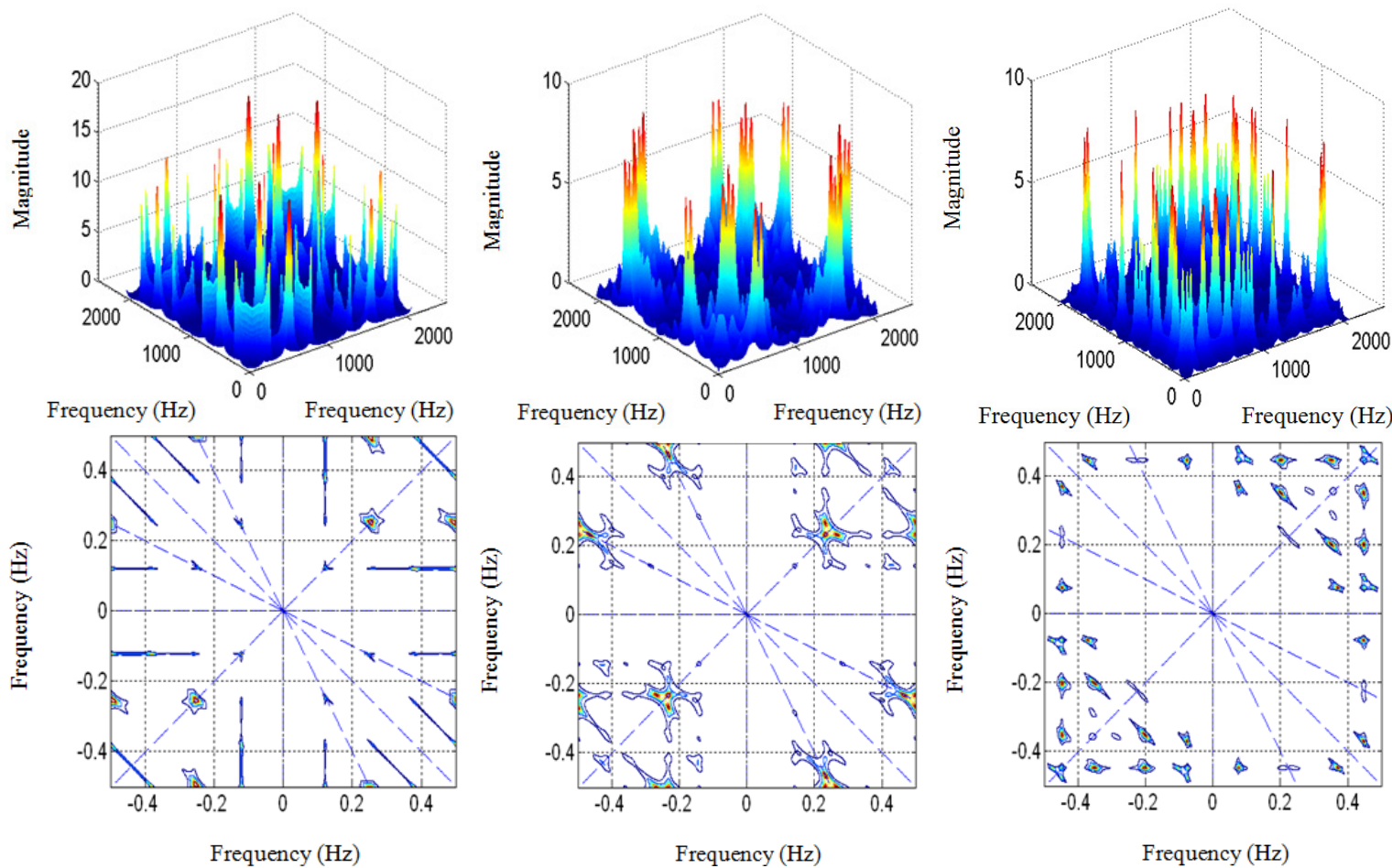

(a)

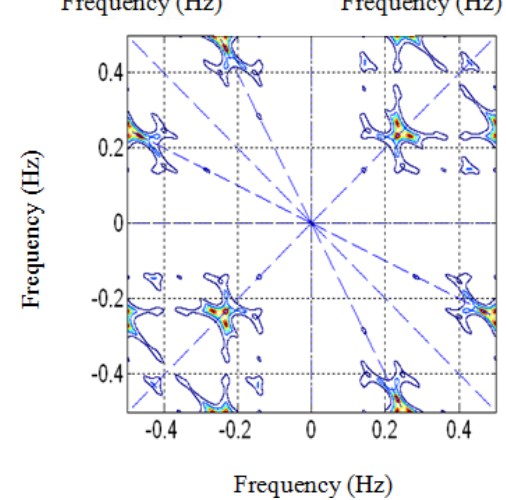

(b)

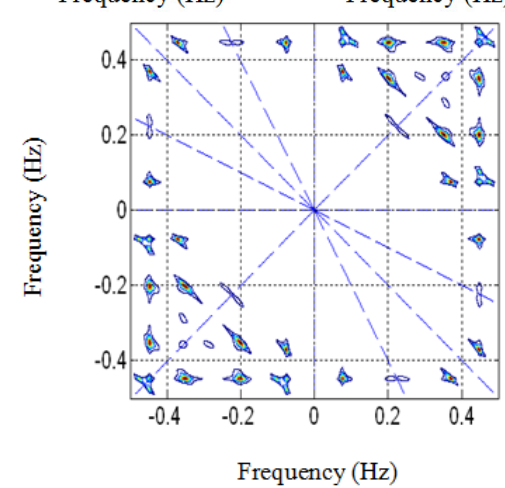

(c)

Figure 4. Bi-spectrum of low-intensity steady state: (a) gas provision $=100 \mathrm{~L} / \mathrm{h}$, one-step air provision $=1400 \mathrm{~L} / \mathrm{h} ;(\mathbf{b})$ gas provision $=100 \mathrm{~L} / \mathrm{h}$, one-step air provision $=1500 \mathrm{~L} / \mathrm{h} ;(\mathbf{c})$ gas provision $=100 \mathrm{~L} / \mathrm{h}$, one-step air provision $=1600 \mathrm{~L} / \mathrm{h}$.
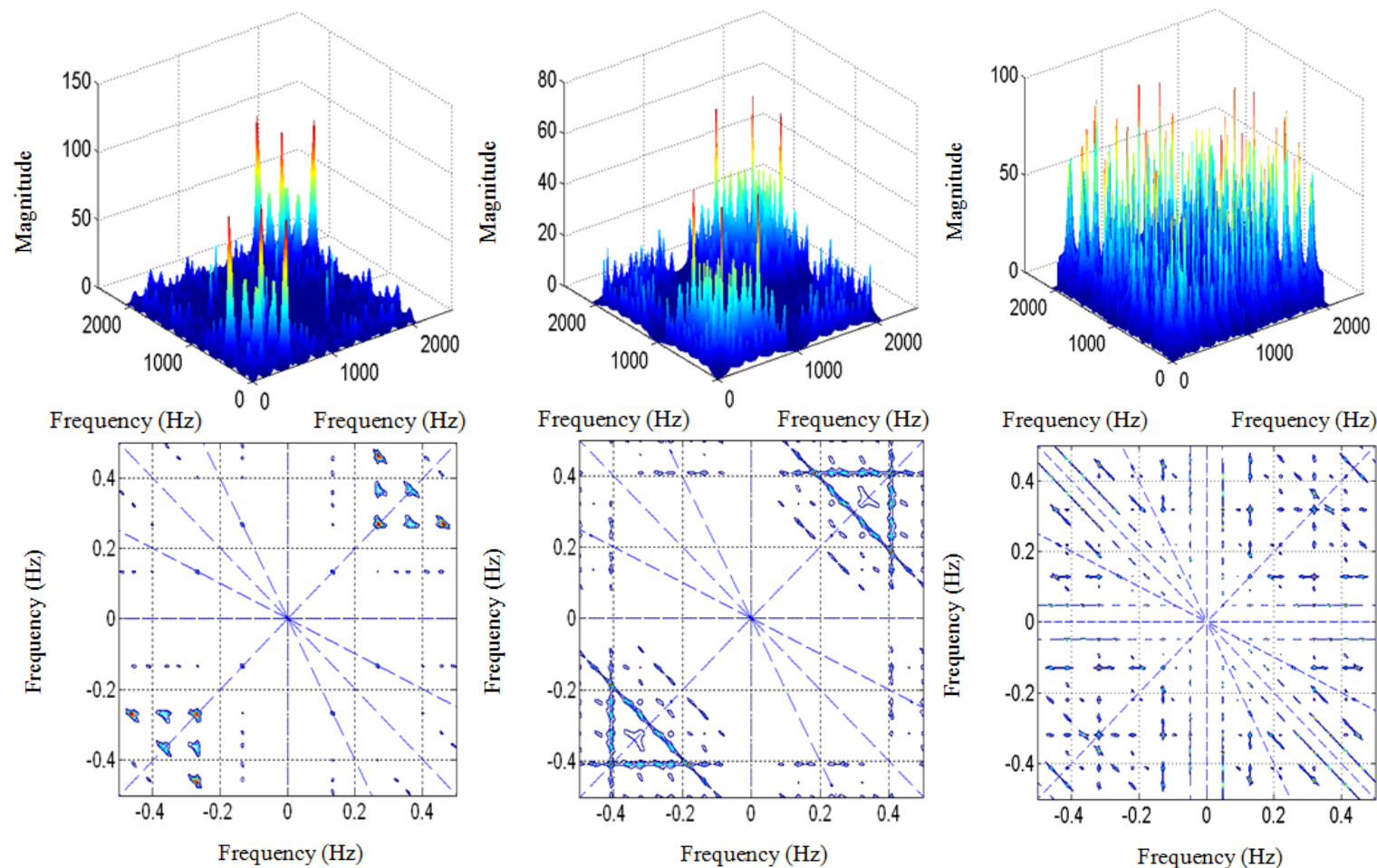

(a)

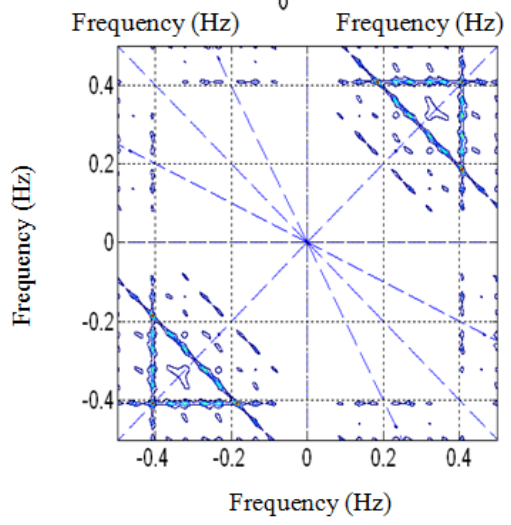

(b)

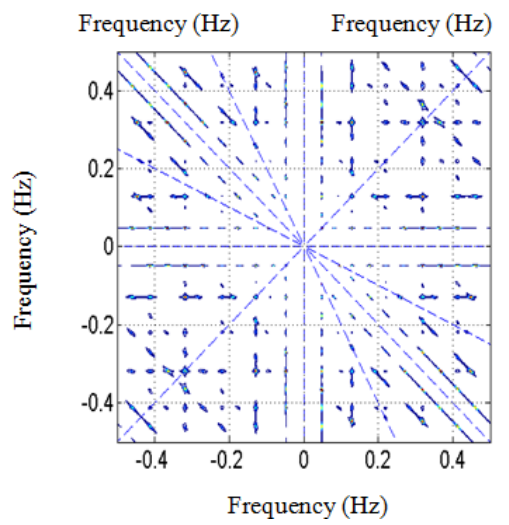

(c)

Figure 5. Bi-spectrum of high-intensity steady state: (a) gas provision $=100 \mathrm{~L} / \mathrm{h}$, one-step air provision $=400 \mathrm{~L} / \mathrm{h} ;(\mathbf{b})$ gas provision $=40 \mathrm{~L} / \mathrm{h}$, one-step air provision $=160 \mathrm{~L} / \mathrm{h} ;(\mathbf{c})$ gas provision $=40 \mathrm{~L} / \mathrm{h}$, one-step air provision $=140 \mathrm{~L} / \mathrm{h}$. 

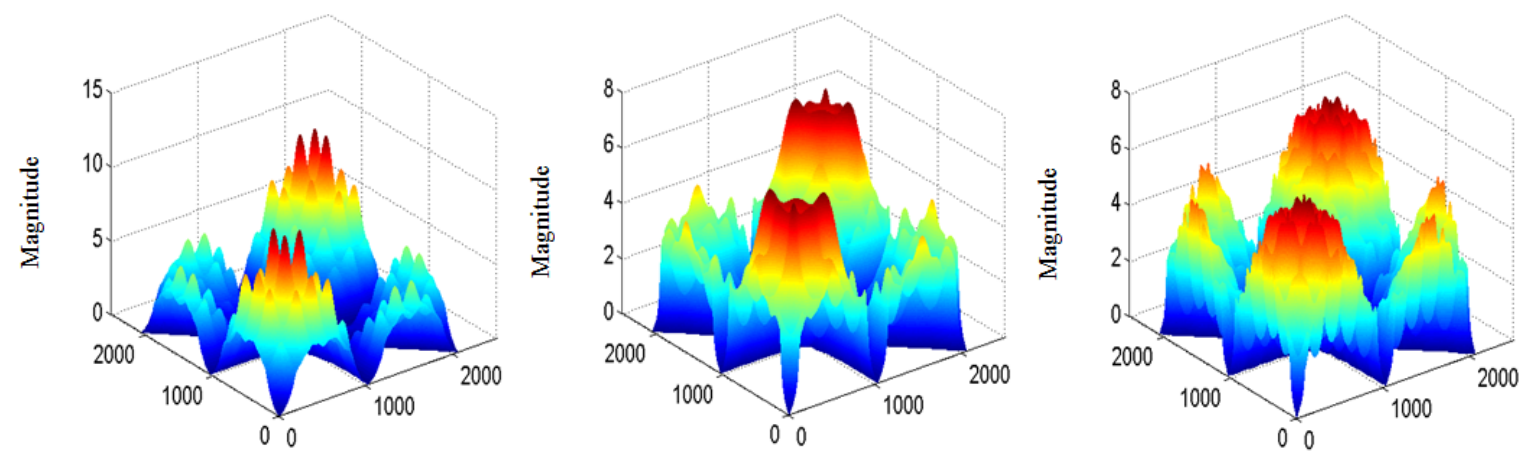

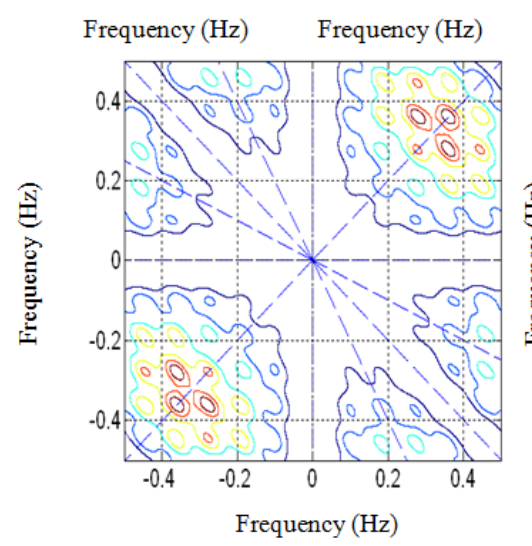

(a)

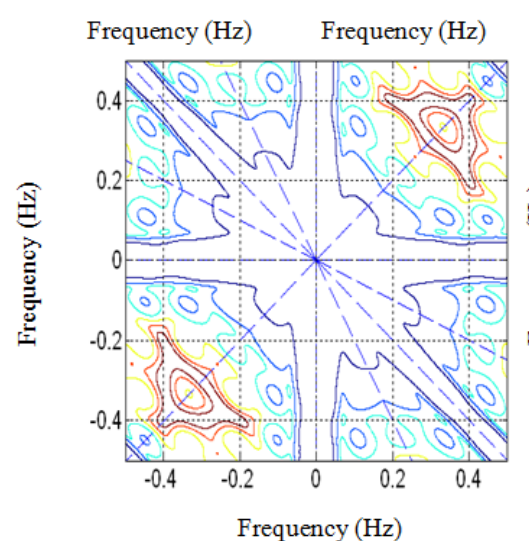

(b)

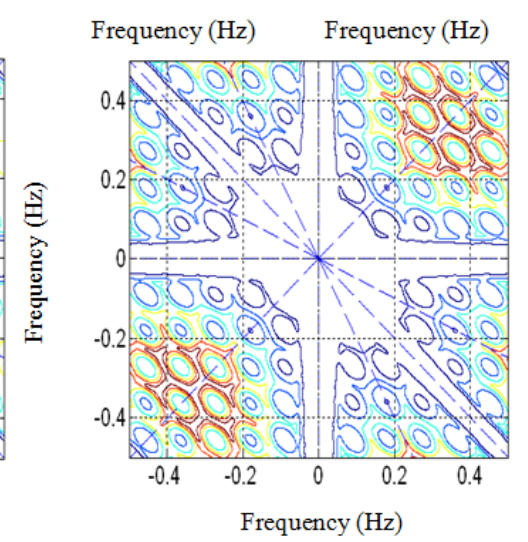

(c)

Figure 6. Bi-spectrum of low-intensity unsteady state: (a) gas provision $=240 \mathrm{~L} / \mathrm{h}$, one-step air provision $=800 \mathrm{~L} / \mathrm{h} ;(\mathbf{b})$ gas provision $=100 \mathrm{~L} / \mathrm{h}$, one-step air provision $=0 \mathrm{~L} / \mathrm{h} ;(\mathbf{c})$ gas provision $=$ $400 \mathrm{~L} / \mathrm{h}$, one-step air provision $=1600 \mathrm{~L} / \mathrm{h}$.

The bi-spectrum of the low-intensity unsteady state displayed in Figure 6 had evident non-linear character, and the bi-spectrum phase remained relatively low, with frequencies concentrated on the extremely high frequencies and the secondarily high frequencies. Bi-spectrums had a clustered distribution and were similar in shape. There were more side lobes existing in the bi-spectrum, with the energy more prominent, and there were also differences in the top peak values. The structure of the bi-spectrum, as seen in Figure 7, was relatively simple. In the whole frequency bi-spectrum, the larger peak values existed sporadically only in high frequency areas, indicating that the gas and air were mixed violently.

The mechanism of non-linearity under different combustion conditions and different stabilities were different so that the bi-spectrum could be distinguished obviously, thus making it relatively easy to distinguish from various combustion conditions. This also sufficed to show that using non-linearity phase coupling in bi-spectrum analysis to diagnose the non-linearity and non-Gaussianity was practical and effective.

Power spectrum is an important signal processing tool. However, as is known by the definition of the power spectrum, the spectral estimation method is essentially the second order statistics of signal analysis; the information obtained merely contains amplitude-frequency characteristics and autocorrelation sequence, and is unable to provide higher-order statistics information. Higher-order statistics in signal processing and system analysis plays a very important role and shows a distinct advantage in signals and systems analysis. For a wave, the phase information can describe the waveform alteration, namely the instantaneous signal phase links to the signal direction. Apparently, phase of the signal stays at a high level under the instability condition, far from the stability condition, while phase remains at a low level under the stability condition. Based on the knowledge of the spectrum, analysis methods reserve the phase information of the signals. The phase information from 
bi-spectrum evaluation is extracted to distinguish the steady state and unsteady state. The theoretical bi-spectrum evaluated from the ARMA model is:

$$
s(n)=s_{r}(n)+i s_{j}(n)
$$

where $s_{r}(n)$ and $s_{j}(n)$, respectively, refer to the real and imaginary part of the bi-spectrum. The phase is defined as:

$$
p(n)=\arctan \left(s_{i}(n) / s_{r}(n)\right) .
$$

The phase and amplitude in the whole frequency bi-spectrum is defined as:

$$
p=\left|\sum_{w 1, w 2} \arctan \left(s_{i}(n) / s_{r}(n)\right)\right|
$$

which gives the statistical phase of $|B x(f 1, f 2)|[20,21]$.
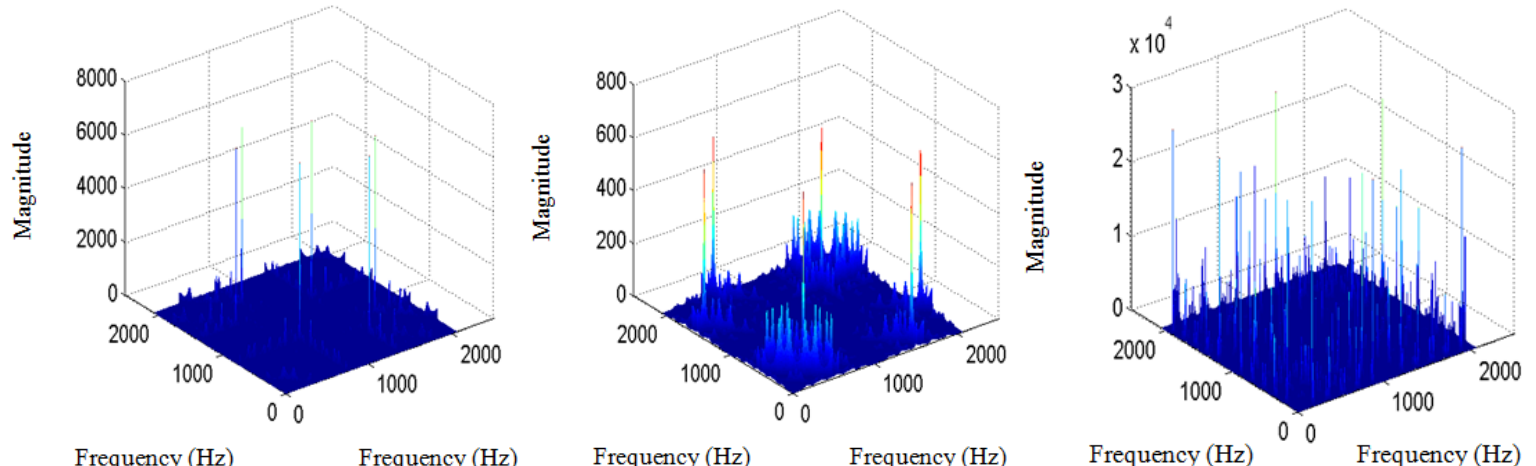

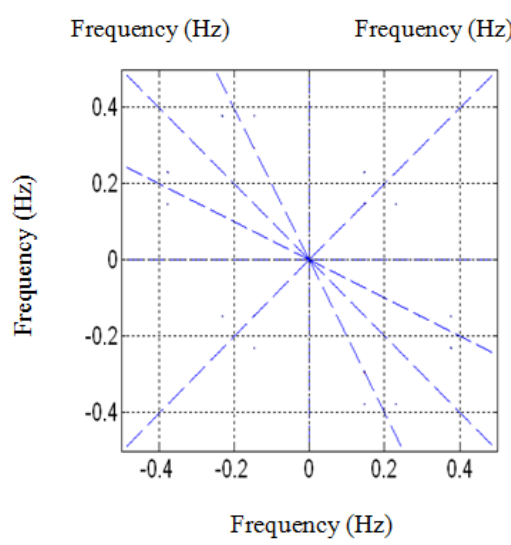

(a)

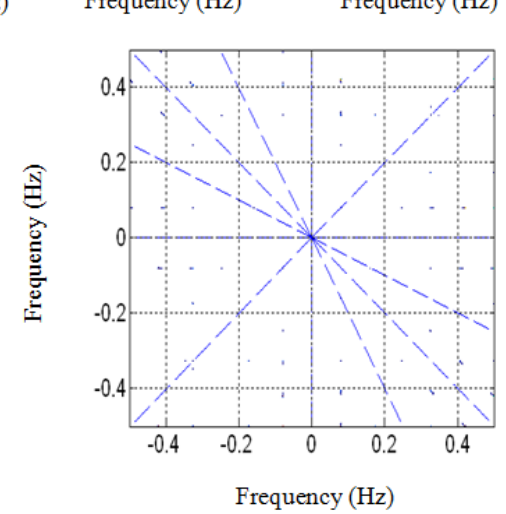

(b)

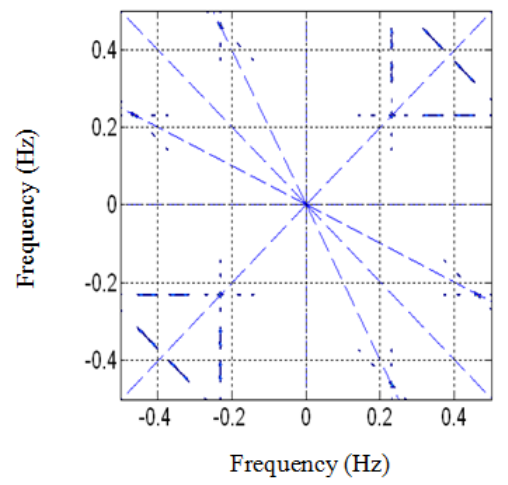

(c)

Figure 7. Bi-spectrum of high-intensity unsteady state: (a) gas provision $=400 \mathrm{~L} / \mathrm{h}$, one-step air provision $=800 \mathrm{~L} / \mathrm{h} ;(\mathbf{b})$ gas provision $=400 \mathrm{~L} / \mathrm{h}$, one-step air provision $=0 \mathrm{~L} / \mathrm{h} ;(\mathbf{c})$ gas provision $=$ $600 \mathrm{~L} / \mathrm{h}$, one-step air provision $=800 \mathrm{~L} / \mathrm{h}$.

Figure 8 shows the relationships of the specific variance, flickering frequency, and bi-spectrum phase with the increasing air/gas ratio. As is shown in Figure 8, there are obvious differences in the specific variance, the flickering frequency, and the bi-spectrum phase, each of them occupying a distinct inter-region. The parameters can reflect the changes of flame stability. 


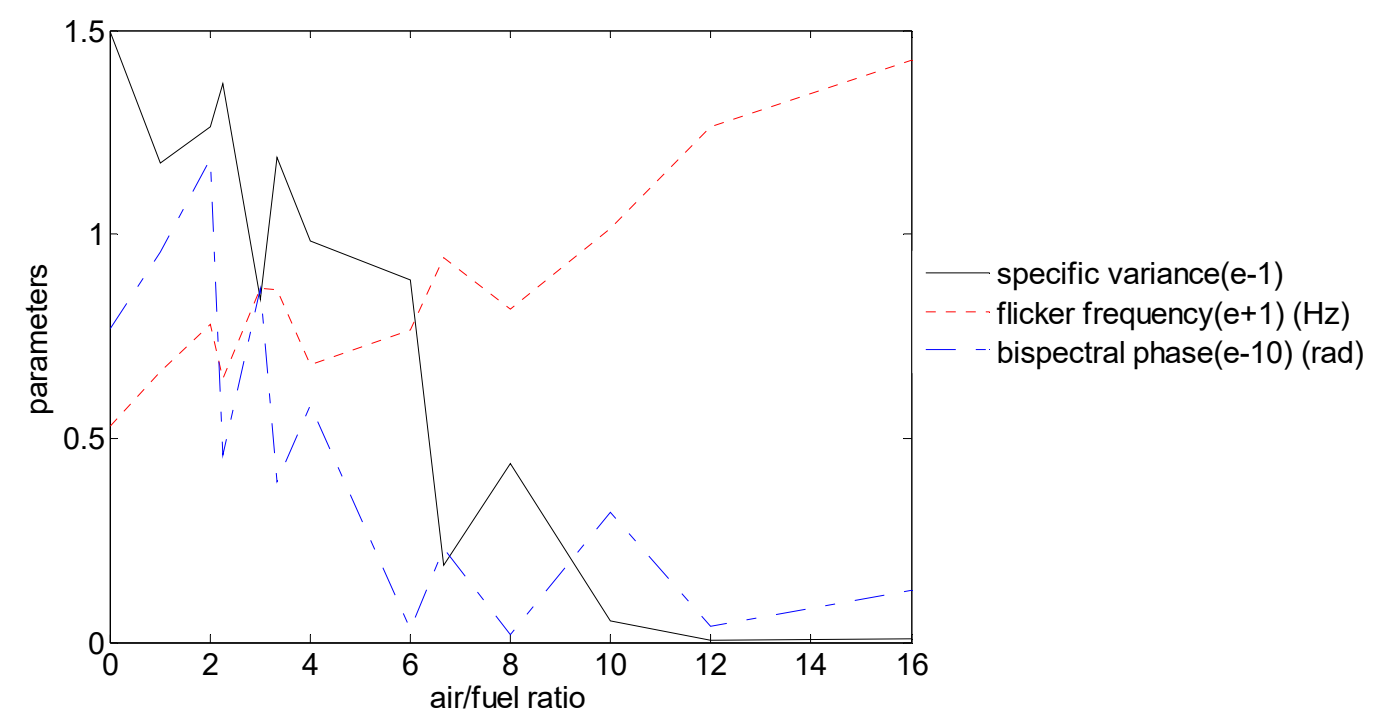

Figure 8. Effect of air/fuel ratio on parameters.

\section{Fuzzy Synthetic Evaluation on Flame Instability}

The relationship between the combustion stability and the characteristic parameters was studied. However, it was difficult to determine which parameter was more reliable. The fuzzy synthetic evaluation method was used to obtain more accurate results.

Factors that reflect the combustion stability include specific variance, flicker frequency, and bi-spectral phase. In this paper, the factor set was $U=(u 1, u 2, u 3)=($ specific variance, flicker frequency, bi-spectral phase).

The determination of weight set was the key to the evaluation. The weight of each factor was given as $A=(a 1, a 2, a 3)=(0.6,0.3,0.1)$.

Evaluation level was computed by the fuzzy judgement. The evaluation set was defined as $V=(v 1, v 2, v 3)=($ stable, transitional stage, unstable). The results of the evaluation model were given as $S=(90,60,30)$.

The most important feature of the fuzzy set theory was fixing the membership as continuous closed interval $(0,1)$ rather than $\{0,1\}$. The membership degrees were applied to solve the contradiction that many other laws are always ignored by the exact demarcation due to the complicated factors. The membership degrees of the judging index were calculated by the membership functions. Based on the analysis of the relationship between the flame instability and the characteristic parameters, the membership degrees of the $i$ th evaluation grade are shown in Figure 9. Number 1 presents the stable condition, while number 0 presents the unstable condition. The line 'stable' describes the level of approaching the stable condition and the line 'unstable' exhibits the probability of being the unstable condition. The threshold $(p 1-p 7)$ of characteristic quantity was set by practical experimental experience. When the numerical value of the characteristic parameters was less-than $\mathrm{p} 1$, the state of the flame combustion was considered highly steady and the membership degrees were $(1,0,0)$. The possibility of being highly unsteady was not clear until the value reached p2. Two critical points came forth with increases in the value. They illustrated the coequal probability of approaching two closer states. As a transitional state, the line 'transitional stage' reached the top in the middle of the threshold. Similarly, the state of the flame combustion was considered highly unsteady and the membership degrees were $(0,0,1)$ when the numerical value of the characteristic parameters was more-than p7. By analyzing the measurement database of this experiment, the formula (5.1) - (5.3) manifested the membership degrees of the judging parameters and the thresholds of membership degrees and characteristic parameters, as seen in Tables 1 and 2. 


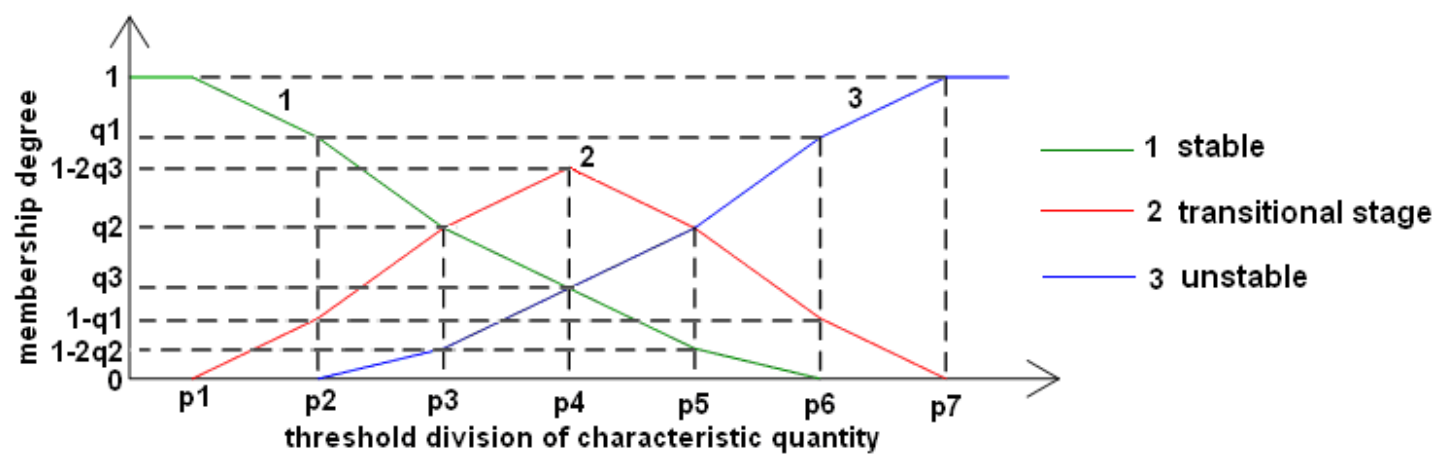

Figure 9. Curves of fuzzy membership function.

Table 1. The threshold division of membership degrees.

\begin{tabular}{cccc}
\hline Threshold & $\boldsymbol{q 1}$ & $\boldsymbol{q} \mathbf{2}$ & $\boldsymbol{q 3}$ \\
\hline value & 0.8 & 0.475 & 0.1 \\
\hline
\end{tabular}

Table 2. The threshold division of characteristic parameters.

\begin{tabular}{cccc}
\hline Parameter & Specific Variance (E-4) & Flicker Frequency (Hz) & Bi-Spectral Phase (E-13) (rad) \\
\hline$p 1$ & 3.53 & 17.58 & 2.322 \\
$p 3$ & 820.92 & 8.45 & 82.97 \\
$p 5$ & 1232.07 & 6.37 & 278.96 \\
$p 7$ & 3737.55 & 2.23 & 1185.7 \\
$p 2(=(p 1+p 3) / 2)$ & 412.23 & 13.01 & 42.64 \\
$p 4(=(p 3+p 5) / 2)$ & 1026.50 & 7.41 & 180.96 \\
$p 6(=(p 5+p 7) / 2)$ & 2484.81 & 4.30 & 732.33 \\
\hline
\end{tabular}

According to the evaluation score calculated, combustion condition class was classified into four grades: highly stable, slightly stable, slightly unstable, and highly unstable. Table 3 shows the combustion condition class and combustion grades.

$$
\begin{gathered}
\delta(x 1)= \begin{cases}1 & x 1<p 1 \\
1-(1-q 1)(x 1-p 1) /(p 2-p 1) & p 1 \leq x 1<p 2 \\
q 1-(q 1-q 2)(x 1-p 2) /(p 3-p 2) & p 2 \leq x 1<p 3 \\
q 2-(q 2-q 3)(x 1-p 3) /(p 4-p 3) & p 3 \leq x 1<p 4 \\
q 3-(q 3+2 q 2-1)(x 1-p 4) /(p 5-p 4) & p 4 \leq x 1<p 5 \\
(1-2 q 2)(p 6-x 1) /(p 6-p 5) & p 5 \leq x 1<p 6 \\
0 & p 6 \leq x 1<p 7 \\
0 & x 1 \geq p 7\end{cases} \\
\delta(x 2)= \begin{cases}0 & x 1<p 1 \\
(1-q 1)(x 2-p 1) /(p 2-p 1) & p 1 \leq x 1<p 2 \\
q 2-(q 2+q 1-1)(p 3-x 2) /(p 3-p 2) & p 2 \leq x 1<p 3 \\
1-2 q 3-(1-2 q 3-q 2)(p 4-x 2) /(p 4-p 3) & p 3 \leq x 1<p 4 \\
(1-2 q 3-q 2)(p 5-x 2) /(p 5-p 4)+q 2 & p 4 \leq x 1<p 5 \\
(q 2+q 1-1)(p 6-x 2) /(p 6-p 5)+1-q 1 & p 5 \leq x 1<p 6 \\
(1-q 1)(p 7-x 2) /(p 7-p 6) & p 6 \leq x 1<p 7 \\
0 & x 1 \geq p 7\end{cases}
\end{gathered}
$$




$$
\delta(x 3)= \begin{cases}0 & x 1<p 1 \\ 0 & p 1 \leq x 1<p 2 \\ (1-2 q 2)(x 3-p 2) /(p 3-p 2) & p 2 \leq x 1<p 3 \\ q 3-(q 3+2 q 2-1)(p 4-x 3) /(p 4-p 3) & p 3 \leq x 1<p 4 \\ q 2-(q 2-q 3)(p 5-x 3) /(p 5-p 4) & p 4 \leq x 1<p 5 \\ q 1-(q 1-q 2)(p 6-x 3) /(p 6-p 5) & p 5 \leq x 1<p 6 \\ 1-(1-q 1)(p 7-x 3) /(p 7-p 6) & p 6 \leq x 1<p 7 \\ 1 & x 1 \geq p 7\end{cases}
$$

Table 3. Combustion condition class.

\begin{tabular}{cccccc}
\hline Score & $>80$ & $\mathbf{6 0 - 8 0}$ & $\mathbf{4 0 - 6 0}$ & $<40$ & Score \\
\hline $\begin{array}{c}\text { combustion } \\
\text { grade }\end{array}$ & highly stable & slightly stable & $\begin{array}{c}\text { slightly } \\
\text { unstable }\end{array}$ & highly unstable & $\begin{array}{c}\text { combustion } \\
\text { grade }\end{array}$ \\
\hline
\end{tabular}

According to the former discussion, practical examples were made to examine the feasibility and reliability of the synthetic evaluation method. Some typical combustion tests were used to investigate the model. The judgment matrix of each condition is constructed as follows:

$$
\begin{aligned}
\left.R\right|_{\text {hightly stable }}= & {\left[\begin{array}{ccc}
1 & 0 & 0 \\
0.8172 & 0.1828 & 0 \\
0.6392 & 0.336 & 0.0247
\end{array}\right] } \\
\left.R\right|_{\text {slightly stable }}= & {\left[\begin{array}{ccc}
0.5714 & 0.3934 & 0.0352 \\
0.0371 & 0.4043 & 0.5586 \\
0.3055 & 0.6219 & 0.0726
\end{array}\right] } \\
\left.R\right|_{\text {slightly unstable }}= & {\left[\begin{array}{ccc}
0.0689 & 0.5978 & 0.3334 \\
0.2905 & 0.6349 & 0.0746 \\
0.3299 & 0.6008 & 0.0693
\end{array}\right] } \\
\left.R\right|_{\text {highly unstable }} & =\left[\begin{array}{ccc}
0 & 0 & 1 \\
0 & 0.1423 & 0.8577 \\
0.02 & 0.31 & 0.67
\end{array}\right]
\end{aligned}
$$

Table 4 gives the flame patterns, their characteristic parameter values, and the evaluation results. The figure presents four different combustion conditions. Their specific variance, flicker frequency, and bi-spectral phase were derived from the data. By computing the evaluation matrix and evaluation score, their combustion grades were calculated. The fuzzy synthetic evaluation of combustion stability based on time-frequency analysis and higher-order statistics was effective. 
Table 4. Evaluation results of typical combustion conditions.

\begin{tabular}{|c|c|c|c|c|}
\hline Condition Number & First & Second & Third & Fourth \\
\hline \multicolumn{5}{|l|}{ flame pattern } \\
\hline specific variance $(\mathrm{E}-4)$ & 3.53 & 699.68 & 1033.54 & 3738.29 \\
\hline flicker frequency $(\mathrm{Hz})$ & 13.41 & 5.84 & 6.16 & 3.70 \\
\hline $\begin{array}{l}\text { bi-spectral phase(E-13) } \\
\text { (rad) }\end{array}$ & 62.59 & 127.26 & 62.14 & 551.05 \\
\hline evaluation matrix & (0.9091 0.08840 .0025$)$ & (0.3845 0.41950 .1960$)$ & (0.1368 0.64120 .2220$)$ & (0.0020 0.07370 .9243$)$ \\
\hline evaluation score & 87.20 & 65.66 & 57.44 & 32.33 \\
\hline combustion grade & highly stable & slightly stable & slightly unstable & highly unstable \\
\hline
\end{tabular}

\section{Conclusions}

A low-cost experimental device was set up to detect flame combustion. A fuzzy synthetic evaluation method based on time-frequency analysis and higher-order statistics was proposed to evaluate flame instability. The following conclusions were reached.

The appropriate operating parameters of the detection circuit were reasonable and a strict signal acquisition process was set up. The results show that the detection of background signals constructs an appropriate working condition for conversion components. It eliminates the direct current signals caused by background radiation, decreases the interference of the background glare from outside, stops the component from coming into the saturation area, and prevents the interference of the background glare from submerging useful signals. The dynamic extension of detection is broadened and the signal to noise ratio of the signals is increased.

Bi-spectrum calculation shows the non-Gaussian and nonlinear characteristics of different combustion conditions. Three-dimensional figures of the bi-spectrum under various combustion conditions revealed the frequency coupling distribution and phase information. Power spectrum and bi-spectrum information of the phase domain (specific variance, flicker frequency, and bi-spectral phase) were derived. The results showed that the flame radiation frequency domain signal was concentrated in the low frequency range $(0$ to $10 \mathrm{~Hz})$. With the increasing air/gas ratio, the flickering frequency increased while the specific variance and the bi-spectrum phase decreased.

By settling of the threshold division of membership degrees and the threshold division of characteristic parameters, the mathematical model of fuzzy comprehensive evaluation and the evaluation index system of combustion stability were established. The computation of four different combustion conditions showed that this fuzzy synthetic evaluation method was effective. Compared to existing time-frequency analysis methods, the time, frequency, and phase domain of the combustion radiation signal were analyzed in this experiment. By analyzing the phase domain signal, the analysis method of the flame radiation signal was expanded. The analytical methods need to be further refined to obtain a specific functional relationship between the combustion state and the flame fluctuation information.

Author Contributions: H.Z. independently completed theoretical calculations, system design, and experimental verification. H.Z. carried out the research and analyzed the numerical data with guidance from M.Z. and X.L. H.Z. prepared the manuscript.

Funding: This research received no external funding.

Conflicts of Interest: The authors declare no conflict of interest. 


\section{References}

1. Yan, B.; Li, B.; Baudoin, E.; Liu, C.; Sun, Z.W.; Li, Z.S.; Bai, X.S.; Aldén, M.; Chen, G.; Mansour, M.S. Structures and Stabilization of Low Calorific Value Gas Turbulent Partially Premixed Flames in a Conical Burner. Exp. Therm. Fluid Sci. 2010, 34, 412-419. [CrossRef]

2. Moore, J.; Risha, G.; Kuo, K.; Zhang, B. Stability of Methane/Oxygen Coaxial Diffusion Flame. In Proceedings of the 39th AIAA/ASME/SAE/ASEE Joint Propulsion Conference and Exhibit, Huntsville, AL, USA, 20-23 July 2003.

3. Komarek, T.; Polifke, W. Impact of Swirl Fluctuation on the Flame Response of a Perfectly Premixed Swirl Burner. J. Eng. Gas Turb. Power 2009, 132, 845-853.

4. Lilleberg, B.; Ertesvag, I.S.; Rian, K.E. Modeling Instabilities in Lean Premixed Turbulent Combustors Using Detailed Chemical Kinetics. Combust. Sci. Technol. 2009, 181, 1107-1122. [CrossRef]

5. Voigt, T.; Habisreuther, P.; Zarzalis, N. Simulation of Vorticity Driven Flame Instability Using a Flame Surface Density Approach Including Markstein Number Effects. In Proceedings of the ASME Turbo Expo 2009: Power for Land, Sea, and Air, Orlando, FL, USA, 8-12 June 2009.

6. Huang, Y.; Yan, Y.; Lu, G.; Reed, A. Online Flicker Measurement of gaseous Flame by Image Processing and Spectral Analysis. Meas. Sci. Technol. 1999, 10, 726-733. [CrossRef]

7. Xu, L.; Yan, Y.; Cornwell, S.; Riley, G. Online Fuel Identification Using Digital Signal Processing and Soft-Computing Techniques. In Proceedings of the IMTC2003, Vail, CO, USA, 20-22 May 2003; pp. 1114-1118.

8. Li, J.; Liu, S.; Lei, J.; Huang, Y.; Li, Z.; Wang, F.; Wang, Z. Compositive Assessment Model of Flame Combustion Stability Based on Grey Theory. In Proceedings of the 2010 International Conference on Optoelectronics and Image Processing, Hainan, China, 11-12 November 2010.

9. Sahu, K.B.; Kundu, A.; Ganguly, R.; Datta, A. Effects of Fuel Type and Equivalence Ratios on the Flickering of Triple Flames. Combust. Flame 2009, 156, 484-493. [CrossRef]

10. Spector, Y.; Jacobson, E. Novel technology for flame and gas detection in the petrochemical industry. Proc. SPIE 1999, 3538, 256-268. [CrossRef]

11. Huang, Y.; Liu, S.; Li, J.; Lei, J. The Identification of the Flame Combustion Stability Based on the Mahalanobis Distance. Appl. Mech. Mater. 2011, 48-49, 1256-1260. [CrossRef]

12. Zhou, G.; Qin, J.; Sun, Y.; Li, Z.; Luo, Z.; Zhou, H. Experimental Detection of Radiative Energy Signal From a Supercharged Marine Boiler and Simulation on its Application in Control of Drum Water Level. Appl. Eng. 2011, 31, 3168-3175.

13. Lv, Z.Z.; Shen, J. Flame Detection and Combustion Diagnosis Technique for Utility Boiler. Boil. Technol. 1997, 5, 8-14. (In Chinese)

14. Ma, J.; Yu, Y.F.; Fan, H.J. Research on Flame Detection and Combustion Diagnosis Based on Spectrum Analysis and with Self Organized Neural Networks. Power Eng. 2004, 6, 2020-2025. (In Chinese)

15. Ao, L.M.; Li, J.H.; Song, X. An Investigation of the Flame Detection Method based on Self-Adaptive Wavelet Conversion. J. Eng. Therm. Energy Power 2006, 21, 594-601. (In Chinese)

16. Chi, T.; Zhang, H. On-line Tracking of Pulverized Coal and Biomass Fuels Through Flame Spectrum Analysis. Chin. J. Sci. Instrum. 2007, 28, 2008-2013. (In Chinese)

17. Shang, H.; Li, J. The Evaluation of Industrial Group's Synergetic Capacity Based on Multi-layer Fuzzy Synthetic Model. J. Converg. Inf. Technol. 2011, 6, 58-66.

18. Huang, S.; Yang, Y. A Study on Evaluation System of Bridge Technical Condition Based on Fuzzy Synthetic Judgment. In Proceedings of the 2011 International Conference on Electric Technology and Civil Engineering, Lushan, China, 22-24 April 2011; pp. 4755-4758.

19. Swami, A.; Mendel, J.M.; Nikias, C.L. Higher-Order Spectral Analysis Toolbox User's Guide; The Math Works Inc.: Natick, MA, USA, 1998.

20. Hickey, D.; Worden, K.; Platten, M.F.; Wright, J.R.; Cooper, J.E. Higher-order Spectra for Identification of Nonlinear Modal Coupling. Mech. Syst. Signal Process. 2009, 23, 1037-1061.

21. Choudhury, M.S.; Shah, S.L.; Thornhill, N.F. Diagnosis of Poor Control-loop Performance Using Higher-order Statistics. Automatica 2004, 40, 1719-1728.

22. Zadeh, L.A. Fuzzy Sets and Fuzzy Information-Granulation Theory: Key Selected Papers by; Beijing Normal University Press: Beijing, China, 2005. 
23. Zang, C.W.; Huang, H.W.; Zhang, Z.X. Forecasting the Strata Condition of a Long Road Tunnel by Using Fuzzy Synthetic Judgment. Int. J. Rock Mech. Min. Sci. 2004, 41, 1-6. [CrossRef]

24. Xu, L.; Yan, Y.; Cornwell, S.; Riley, G. On-line Fuel Identification Using Digital Signal Processing and Fuzzy Inference Techniques. IEEE Trans. Instrum. Meas. 2004, 53, 1316-1320. [CrossRef]

25. Xu, L.; Yan, Y.; Cornwell, S.; Riley, G. Online Fuel Tracking by Combining Principal Component Analysis and Neural Network Techniques. IEEE Trans. Instrum. Meas. 2005, 54, 1640-1645. [CrossRef]

26. Hua, Y.P.; Zou, Y.; Lv, Z.Z. A Comprehensive Survey of Flame Detection Techniques Used in Modern Coal-fired Utility Boilers. J. Eng. Therm. Energy Power 2001, 16, 1-5. (In Chinese)

27. Cai, G.; Zhu, M. Moment Inequality and Complete Convergence of $\rho$ Mixing Sequences. J. Chongqing Univ. 2001, 29, 44-47. (In Chinese)

(C) 2019 by the authors. Licensee MDPI, Basel, Switzerland. This article is an open access article distributed under the terms and conditions of the Creative Commons Attribution (CC BY) license (http:/ / creativecommons.org/licenses/by/4.0/). 